\title{
Phase field simulation of interatomic potentials for double phase competition during early stage precipitation
}

\author{
DONG WeiPing ${ }^{*}$, WANG YongXin, YANG Kun, CHEN Zheng \& LU YanLi \\ State Key Laboratory of Solidification Processing, Northwestern Polytechnical University, Xi'an 710072, China
}

Received April 20, 2010; accepted January 10, 2011

\begin{abstract}
Phase field model was employed to study the variations of interatomic potentials of $\mathrm{Ni}_{3} \mathrm{Al}\left(\mathrm{Ll}_{2}\right.$ phase $)$ and $\mathrm{Ni}_{3} \mathrm{~V}\left(\mathrm{DO}_{22}\right.$ phase $)$ as a function of temperature and concentration. The long-range order (LRO) parameter related interatomic potentials equations formulated by Khachaturyan were utilized to establish the inversion equations for $\mathrm{L}_{2}$ and $\mathrm{DO}_{22}$ phases, with which interatomic potentials could be calculated. The interatomic potentials of $\mathrm{Ni}-\mathrm{Al}$ and $\mathrm{Ni}-\mathrm{V}$ exhibited approximately linear increases and decreases, individually, with enhanced Al concentration. Substituting the inverted interatomic potentials into the microscopic phase field equations led to three cases of precipitation sequence: the $\mathrm{DO}_{22}$ phase preceded $\mathrm{L} 1_{2}$ phase precipitating at the interatomic potentials of Ni-V > Ni-Al; the vice cases; and two phases precipitated simultaneously at interatomic potentials of $\mathrm{Ni}-\mathrm{V}$ and $\mathrm{Ni}-\mathrm{Al}$ were equal.
\end{abstract}

phase field model, $\mathrm{L1}_{2}$ and $\mathrm{DO}_{22}$ phases, interatomic potentials, double phase competition

Citation: Dong W P, Wang Y X, Yang K, et al. Phase field simulation of interatomic potentials for double phase competition during early stage precipitation. Chinese Sci Bull, 2011, 56: 2055-2060, doi: 10.1007/s11434-011-4407-1

Phase transformation involves atomic diffusion and clustering, which occurs under potential energy produced external changes. During solid-state phase transformation, the product phase often nucleates and forms at the interface of the parent phase. Multiple-phase mixtures are likely coexisting in multiple component alloys, which precipitate sequentially and form first or second-phase precipitates during the early stages of precipitation [1]. Ni-Al-V alloy, which two ordered phases are produced at different temperatures and composition, is a typical alloy exhibiting this behavior. When Ni-Al-V alloy is quenched into a miscibility gap, a coherent mixture of disordered face-centered cubic (FCC) matrix, $\mathrm{DO}_{22}$ phase and $\mathrm{L1}_{2}$ phase develop. Because of the differences in interatomic potentials and nucleation mechanism of these two phases, their precipitation sequences will differ accordingly. Lattice mismatch between $\mathrm{L}_{2}$ and $\mathrm{DO}_{22}$ is minimal, and their atomic arrangement is similar, thus they can coexist at lattice interfaces.

*Corresponding author (email: penny1688@gmail.com)
Pareige et al. [2] utilized the Monte Carlo method to study early stage isothermal aging of ternary $\mathrm{Ni}_{78.3} \mathrm{Al}_{6.6} \mathrm{~V}_{15.2}$ alloys at $800^{\circ} \mathrm{C}$, and they discovered that a dynamic transformation from $\mathrm{FCC}$ to $\mathrm{FCC}+\mathrm{L}_{2}+\mathrm{DO}_{22}$ occurred. The precipitation of the $\mathrm{L}_{2}$ phase $\left(\mathrm{Ni}_{3} \mathrm{Al}\right)$ was found preceding that of the $\mathrm{DO}_{22}$ phase $\left(\mathrm{Ni}_{3} \mathrm{~V}\right)$, and these two phases could coexist. This phenomenon had also been interpreted by phase field model by Poduri et al. [3] studying the ordering of the pseudo $\mathrm{Ni}_{3} \mathrm{Al}-\mathrm{Ni}_{3} \mathrm{~V}$ binary system. Three-dimensional atomic probe analysis and microscopic mean field numeric calculations of the transformation of the ternary $\mathrm{Ni}_{78.5} \mathrm{Al}_{7} \mathrm{~V}_{14.5}$ alloy under isothermal aging at $800^{\circ} \mathrm{C}$ yielded similar observations [4]. Li et al. [5,6] and Hou et al. [7] employed microscopic phase field theory to explore the coexistence and creation process of the two phases, and the results showed that the precipitation sequence were close related to $\mathrm{Al}$ atomic concentration.

The properties of an intermetallic compound lie on lattice structure and interatomic potentials which is capable to be calculated with a function. Because of the complexity of a 
lattice structure, the most straightforward approximated form of the interatomic potentials is to break the lattice structure into atomic pair potentials. The study concerning interatomic potentials-composition relation which is closely related with each other has been draw attention from both experimental and theoretical point of view. The concentration dependence properties of an intermetallic compound are predominantly demonstrated by concentration dependent interatomic potentials. $\mathrm{Hu}$ et al. [8] obtained phase transformation samples of TiAl alloy after continuous cooling by quenching and air cooling, and showed that the phase transformation microstructures were related to alloy composition. Li et al. [9] studied alloy phases using X-ray and electron microscopy methods, and demonstrated that the volume fractions of $\gamma^{\prime}$ and $\beta$ phases depended on double phase competition which was explained by nucleation dynamics and dendritic growth. Liu et al. [10] performed a super-cooling conditions experiment, illuminating the existence of phase competition in growth processes and multiple phase nucleations by which the formation of a second-phase between equilibrium and metastable phases was explained. Kim et al. [11] investigated interface properties as well as interfacial-misfit energies using modified analytic embedded-atom method (MEAM) potential energies, which were consistent with first principles prediction under the same conditions. Pasianot et al. [12] developed an interatomic potentials method for evolutional microstructure and point defects of the $\mathrm{Fe}-\mathrm{Cu}$ system based on the embedded atom method (EAM) method. Liubich et al. [13] discussed the relationship between double phase competition and composition with the concentration wave (CW) method, and showed that interaction parameters of the disordered A2 and stable B2 phases depending on atomic concentration.

In this study, phase field theory and the $\mathrm{CW}$ method were combined to calculate the concentration-dependence of atomic interactions. The precipitation sequences of $\mathrm{DO}_{22}$ and $\mathrm{L}_{2}$ competitive system of $\mathrm{Ni}_{75} \mathrm{Al}_{x} \mathrm{~V}_{25-x}$ alloy were evaluated using these interatomic potentials.

\section{Theoretical model}

\subsection{Microscopic phase field model}

The phase field dynamic equation is based on the Onsager and Ginzburg-Landau theory, which is written as

$$
\frac{\partial P(\vec{r}, t)}{\partial t}=\sum_{r^{\prime}} L\left(\vec{r}-\vec{r}^{\prime}\right) \frac{\delta F}{\delta P\left(\vec{r}^{\prime}, t\right)} .
$$

This describes the atomic configuration and precipitation sequence of ordered phases using the occupation probability $P(\vec{r}, t)$ at the crystal lattice site $\vec{r}$, and the time $t$, whose change rate is proportional to the variation in free energy. $L\left(\vec{r}-\overrightarrow{r^{\prime}}\right)$ is a constant related to exchange probabilities of a pair of atoms at lattice site $r$ and $r^{\prime}$ per unit time. $F$ is the free energy. For the ternary system, which the author only considers the response of the integrated lattice to atomic diffusion, the occupation probabilities satisfy

$$
P_{A}(\vec{r}, t)+P_{B}(\vec{r}, t)+P_{C}(\vec{r}, t)=1,
$$

where the subscripts $A, B$ and $C$ designate three kinds of atoms. According to eq. (2), the following equations can be obtained:

$$
\left\{\begin{aligned}
\frac{\mathrm{d} P_{A}(\vec{r}, t)}{\mathrm{d} t}= & \frac{1}{k_{\mathrm{B}} T} \sum_{r^{\prime}}\left[L_{A A}\left(\vec{r}-\vec{r}^{\prime}\right) \frac{\partial F}{\partial P_{A}\left(\vec{r}^{\prime}, t\right)}\right. \\
& \left.+L_{A B}\left(\vec{r}-\vec{r}^{\prime}\right) \frac{\partial F}{\partial P_{B}\left(\vec{r}^{\prime}, t\right)}\right]+\zeta_{1}(\vec{r}, t), \\
\frac{\mathrm{d} P_{B}(\vec{r}, t)}{\mathrm{d} t}= & \frac{1}{k_{\mathrm{B}} T} \sum_{r^{\prime}}\left[L_{B B}\left(\vec{r}-\vec{r}^{\prime}\right) \frac{\partial F}{\partial P_{B}\left(\vec{r}^{\prime}, t\right)}\right. \\
& \left.+L_{A B}\left(\vec{r}-\vec{r}^{\prime}\right) \frac{\partial F}{\partial P_{A}\left(\vec{r}^{\prime}, t\right)}\right]+\zeta_{2}(\vec{r}, t) .
\end{aligned}\right.
$$

In the mean-field approximation, the free energy for the ternary system is given by

$$
\begin{aligned}
F= & -\frac{1}{2} \sum_{r} \sum_{r^{\prime}}\left[\left(-V_{A B}\left(\vec{r}-\vec{r}^{\prime}\right)+V_{B C}\left(\vec{r}-\vec{r}^{\prime}\right)\right.\right. \\
& \left.+V_{A C}\left(\vec{r}-\vec{r}^{\prime}\right)\right) P_{A}(\vec{r}) P_{B}\left(\vec{r}^{\prime}\right)+V_{A C}\left(\vec{r}-\vec{r}^{\prime}\right) P_{A}(\vec{r}) P_{A}\left(\vec{r}^{\prime}\right) \\
& \left.+V_{B C}\left(\vec{r}-\vec{r}^{\prime}\right) P_{B}(\vec{r}) P_{B}\left(\vec{r}^{\prime}\right)\right] \\
& +k_{B} T \sum_{r^{\prime}}\left[P_{A}(\vec{r}) \ln \left(P_{A}(\vec{r})\right)+P_{B}(\vec{r}) \ln \left(P_{B}(\vec{r})\right)\right. \\
& \left.+\left(1-P_{A}(\vec{r})-P_{B}(\vec{r})\right) \ln \left(1-P_{A}(\vec{r})-P_{B}(\vec{r})\right)\right]
\end{aligned}
$$

where the first sum represents the chemical energy, the second represents the thermal dynamic energy of the system, and $k_{B}$ is the Boltzmann constant, $T$ is temperature. The effective interaction energy $V_{a b}\left(\vec{r}-\vec{r}^{\prime}\right)$ is given as

$$
V_{a b}\left(r-r^{\prime}\right)=W_{a a}\left(r-r^{\prime}\right)+W_{b b}\left(r-r^{\prime}\right)-2 W_{a b}\left(r-r^{\prime}\right),
$$

where $W_{a a}, W_{b b}$ and $W_{a b}$ are the pairwise potentials between $a-a, b-b$, and $a-b$, respectively, and $V_{a b}$ corresponds to the interaction energy. The four nearest neighbor interaction energies are adopted to maintain reliability of the simulation. The first-nearest $\left(V_{a b}{ }^{1}\right)$, second-nearest $\left(V_{a b}{ }^{2}\right)$, third-nearest $\left(V_{a b}{ }^{3}\right)$ and fourth-nearest $\left(V_{a b}{ }^{4}\right)$ interaction energies are substituted into the FCC reciprocal space:

$$
\begin{aligned}
V_{a b}(\vec{k})= & 4 V_{a b}^{1}(\cos \pi h \cdot \cos \pi k+\cos \pi h \cdot \cos \pi l \\
& +\cos \pi k \cdot \cos \pi l) \\
& +2 V_{a b}^{2}(\cos 2 \pi h+\cos 2 \pi k+\cos 2 \pi l) \\
& +8 V_{a b}^{3}(\cos 2 \pi h \cdot \cos \pi k \cdot \cos \pi l+\cos \pi h \\
& \cdot \cos 2 \pi k \cdot \cos \pi l+\cos \pi h \cdot \cos \pi k \cdot \cos 2 \pi l \\
& +4 V_{a b}^{4}(\cos 2 \pi h \cdot \cos 2 \pi k+\cos 2 \pi h \cdot \cos 2 \pi l \\
& +\cos 2 \pi k \cdot \cos 2 \pi l),
\end{aligned}
$$


where $k, h$ and $l$ are reciprocal lattice sites obtained from

$$
\vec{k}=\left(k_{x}, k_{y}, k_{z}\right)=h \vec{a}_{1}^{*}+k \vec{a}_{2}^{*}+l \vec{a}_{3}^{*},
$$

where $\vec{a}_{1}^{*}, \vec{a}_{2}^{*}, \vec{a}_{3}^{*}$ are the unit reciprocal lattice vectors of the FCC structure along three directions.

\subsection{Interatomic potentials}

Equations for interatomic potentials evaluation were first proposed by Khachaturyan [14]. Their relation to the occupation probability of solute atoms ( $B$ atoms) and free energy were determined based on $\mathrm{CW}$ equations as follows:

$$
\begin{aligned}
& c\left(1+\sum_{s=1}^{t-1} \eta_{s} E_{s}(r)\right)= \\
& {\left[\exp \left(\frac{-\mu+c \cdot V(0)-c \cdot V\left(k_{s}\right) \cdot \eta_{s} E_{s}(r)}{k_{B} T}\right)+1\right]^{-1},}
\end{aligned}
$$

where $c$ is the atomic concentration of solute $B, \eta_{s}$ is the LRO parameter, $E_{s}(r)$ is a function associated with lattice symmetry, $\mu$ is the chemical potential, $t-1$ indicates the number of non-zero vector $k_{s}$ in the superlattice structure, and $V(0)$ the potential energy of disordered atoms, $V\left(k_{s}\right)$ is the potential energy of ordered atoms.

The occupation probability and energy of the $\mathrm{L}_{2}$ phase can be related according to eq. (8):

$$
\begin{gathered}
c(1-\eta)=\left[\exp \left(\frac{-\mu+c \cdot V(0)-c \cdot V\left(k_{0}\right) \cdot \eta}{k_{B} T}\right)+1\right]^{-1}, \\
c(1+3 \cdot \eta)=\left[\exp \left(\frac{-\mu+c \cdot V(0)+3 \cdot c \cdot V\left(k_{0}\right) \cdot \eta}{k_{B} T}\right)+1\right]^{-1} .
\end{gathered}
$$

The combination of eqs. (9) and (10) and subsequent simplification leads to an equation relating $V_{a b}{ }^{1}$ and the LRO parameter:

$$
\frac{-4 V_{\mathrm{ab}}^{1} \cdot \eta}{k_{B} T}=\ln \frac{(1-\eta)[1-c(1+3 \cdot \eta)]}{(1+3 \cdot \eta)[1-c(1-\eta)]} .
$$

This equation can be used to calculate $V_{a b}{ }^{1}$ of the $\mathrm{L}_{2}$ phase at different temperatures and atomic concentrations.

In a similar manner, the occupation probability and energy of the $\mathrm{DO}_{22}$ phase can be related based on eq. (8):

$$
\begin{aligned}
& c\left(1-\eta_{1}\right)=\left[\exp \left(\frac{-u+c \cdot\left(V(0)-V\left(k_{0}\right) \cdot \eta_{1}\right)}{k_{B} T}\right)+1\right]^{-1}, \\
& c\left(1+\eta_{1}+2 \eta_{2}\right)= \\
& {\left[\exp \left(\frac{-u+c \cdot\left(V(0)+V\left(k_{0}\right) \cdot \eta_{1}+2 V\left(k_{1}\right) \cdot \eta_{2}\right)}{k_{B} T}\right)+1\right]^{-1},}
\end{aligned}
$$

$$
\begin{aligned}
& c\left(1+\eta_{1}-2 \eta_{2}\right)= \\
& {\left[\exp \left(\frac{-u+c \cdot\left(V(0)+V\left(k_{0}\right) \cdot \eta_{1}-2 V\left(k_{1}\right) \cdot \eta_{2}\right)}{k_{B} T}\right)+1\right]^{-1} .}
\end{aligned}
$$

An equation relating $V_{a b}{ }^{l}$ to the LRO parameters can be obtained by combining eqs. (12)-(14), followed by simplification:

$$
\frac{-2 \mathrm{~V}_{\mathrm{ab}}^{1}}{k_{B} T}\left(\eta_{1}+\eta_{2}\right)=\ln \frac{\left[1-c\left(1+\eta_{1}+2 \eta_{2}\right)\right]\left(1-\eta_{1}\right)}{\left[1-c\left(1-\eta_{1}\right)\right]\left(1+\eta_{1}+2 \eta_{2}\right)} .
$$

Similarly, the first nearest neighbor interatomic potentials $V_{a b}{ }^{1}$ of the $\mathrm{DO}_{22}$ phase under varying temperature and atomic concentration can be calculated using eq. (15).

These equations were applied to the $\mathrm{Ni}_{75} \mathrm{Al}_{x} \mathrm{~V}_{25-x}$ alloy study. Such equations overcome the deficiencies of conventional models which ignore the impact of temperatures and concentrations using only fixed interatomic potentials in a computation.

\section{Computational results}

\subsection{Interatomic potentials}

$V_{a b}{ }^{1}$ values for the $\mathrm{L}_{2}$ phase $(\mathrm{Ni}-\mathrm{Al})$ with different temperatures and concentrations are obtained by solving eq. (11). Interatomic potentials are measured at a constant temperature of $1000 \mathrm{~K}$ with different $\mathrm{Al}\left(\mathrm{L}_{2}\right)$ or $\mathrm{V}\left(\mathrm{DO}_{22}\right)$ concentrations, and plot against the long-range order (LRO) parameter (Figure 1(a) is for $\mathrm{L1}_{2}$, and Figure 1(b) for $\mathrm{DO}_{22}$ ). The curves increase gradually at comparatively low LRO parameter, but increase dramatically when the LRO parameter approaches one. One likely explanation for this phenomena is that the calculation of interatomic potentials is based on the integrated nucleated $\mathrm{L}_{2}$ phase from which the interatomic potentials are obtained by optimizing the mean interatomic potentials at the LRO parameter range of 0.95-0.99. The Ni-Al interatomic potentials increase with Al concentration ranging from 3-9 at.\% (Figure 1(a)). For the $\mathrm{DO}_{22}$ phase, the $\mathrm{Ni}-\mathrm{V}$ interatomic potentials increase with enhanced LRO parameter and enhanced $\mathrm{V}$ level (16-22 at.\%). Similar to the $\mathrm{L1}_{2}$ phase, the calculation of interatomic potentials for $\mathrm{DO}_{22}$ phase is based on the integrated nucleated $\mathrm{DO}_{22}$ phase from which the interatomic potentials are obtained by optimizing the mean interatomic potentials at the LRO parameter range of 0.95-0.99.

With the temperature holding as a constant at $1000 \mathrm{~K}$, the interatomic potentials obtained at different atomic concentrations are listed by Table $1 . V_{a b}{ }^{1}$ for $\mathrm{L}_{2}$ phase increase from 112.89 to $128.60 \mathrm{meV}$ as $\mathrm{Al}$ concentrations increase from 2 at. $\%$ to 14 at. $\%$, While $V_{a b}{ }^{1}$ for $\mathrm{DO}_{22}$ phase decrease from 132.20 to $100.51 \mathrm{meV}$ as the $\mathrm{V}$ concentrations decrease from 23 at. $\%$ to 11 at.\%, which indicates that interatomic potentials of $\mathrm{L}_{2}$ will increase with enhanced $\mathrm{Al}$ level 

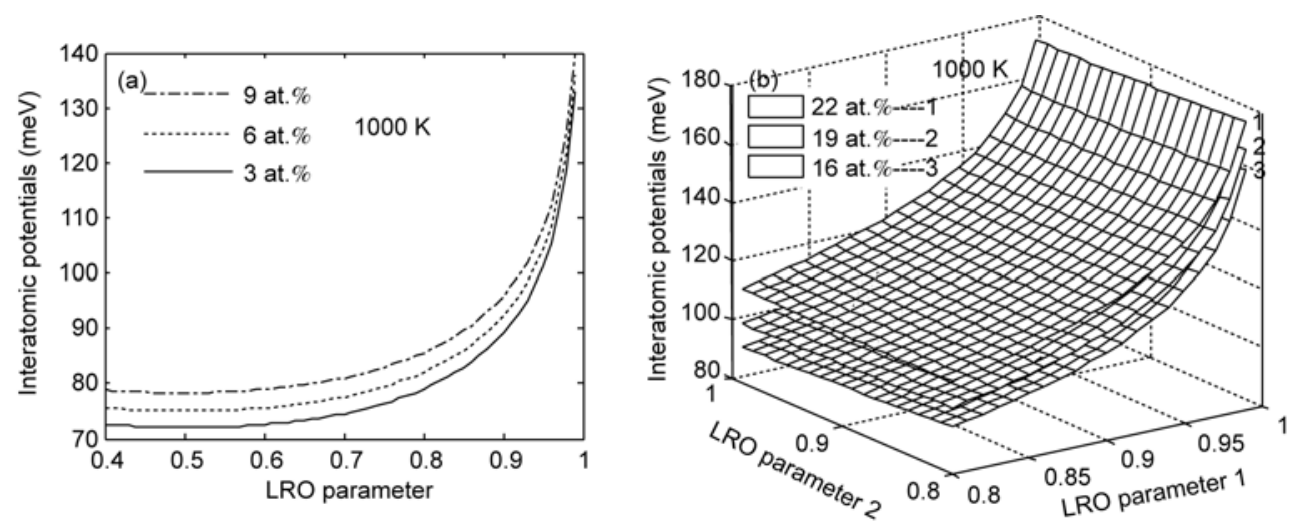

Figure 1 Interatomic potentials versus $\mathrm{LRO}$ parameter for (a) $\mathrm{Ni}-\mathrm{Al}$ and (b) $\mathrm{Ni}-\mathrm{V}$, at different $\mathrm{Al}$ concentrations.

Table 1 Variation of interatomic potentials with concentration for $\mathrm{L}_{2}$ and $\mathrm{DO}_{22}$ phases

\begin{tabular}{cccc}
\hline$c_{\mathrm{Al}}($ at.\% $)$ & $c_{\mathrm{V}}($ at.\% $)$ & $V_{a b \mathrm{~L} 1_{2}}^{1}(\mathrm{meV})$ & $V_{a b \mathrm{DO}_{22}}^{1}(\mathrm{meV})$ \\
\hline 2 & 23 & 112.89 & 132.20 \\
6 & 19 & 116.99 & 116.52 \\
10 & 15 & 122.04 & 107.19 \\
14 & 11 & 128.60 & 100.51 \\
\hline
\end{tabular}

but decrease with enhanced $\mathrm{V}$ concentration in $\mathrm{Ni}_{75} \mathrm{Al}_{x} \mathrm{~V}_{25-x}$ alloy. As a contrast, the interatomic potentials of $\mathrm{DO}_{22}$ phase will decrease with enhanced $\mathrm{Al}$ level but increase with enhanced V level. The computed values of this work approach to the results reported by Chen et al. [3, 4] which $V_{a b}{ }^{1}=122.30$ and $107.2 \mathrm{meV}$ for respective $\mathrm{Ll}_{2}$ and $\mathrm{DO}_{22}$ phases for 10 at.\% $\mathrm{Al}$ alloy, which inverses verified the credibility of the model we employed. We then substitute the computed interatomic potentials into the microscopic phase field for further simulation.

\subsection{The microstructure morphology and volume frac- tion}

The precipitation sequence is closely related to the interatomic potentials. In this study, we employ the evolutional microstructure morphology and volume fraction of the two competitive $\mathrm{L}_{2}$ and $\mathrm{DO}_{22}$ phases, at the early stage of precipitation for different $\mathrm{Al}$ concentrations, exploring precipitation sequence-interatomic potentials relation. At a constant temperature of $1000 \mathrm{~K}$, the evolutional microstructure morphology of the $\mathrm{Ll}_{2}$ and $\mathrm{DO}_{22}$ phases of $\mathrm{Ni}_{75} \mathrm{Al}_{x} \mathrm{~V}_{25-x}$ alloy with enhanced $\mathrm{Al}$ level is illustrated by Figure 2 . The $\mathrm{DO}_{22}$ phase precipitates first at a 4 at. $\% \mathrm{Al}$ alloy (Figure 2(a)), and $\mathrm{L1}_{2}$ phase precipitates gradually at the boundary of $\mathrm{DO}_{22}$ phase with time proceeds. $\mathrm{L}_{2}$ and $\mathrm{DO}_{22}$ phases precipitate simultaneously at a 6 at.\% Al alloy (Figure 2(b)), and the two phases grow competitively with time proceeds. $\mathrm{L}_{2}$ phase precipitates first and the $\mathrm{DO}_{22}$ phase precipitates later at the phase boundary of $\mathrm{L1}_{2}$ at 8 at.\% $\mathrm{Al}$ alloy, which is shown as Figure 2(c) and (f).

The evolutional curves of the volume fractions for $\mathrm{L}_{2}$ phase and $\mathrm{DO}_{22}$ phase in a $\mathrm{Ni}_{75} \mathrm{Al}_{x} \mathrm{~V}_{25-x}$ alloy aging at 1000 $\mathrm{K}$ are presented in Figure 3. As to a 4 at.\% $\mathrm{Al}$ alloy, the system at the beginning which is known as the gestation stage (incubation period ) (Figure 3(a)) is disordered, and the volume fraction for both $\mathrm{L}_{2}$ phase and $\mathrm{DO}_{22}$ phase are almost zero. With time proceeds, the volume fraction of $\mathrm{DO}_{22}$ phase increases rapidly, and then a steadily decaying decrease till the ordered stage, and attains equilibrium gradually. The volume fraction for $\mathrm{L}_{2}$ phase increases after the formation of the $\mathrm{DO}_{22}$ phase, which indicates that the $\mathrm{L}_{2}$ phase has a longer gestation stage than $\mathrm{DO}_{22}$. This result is in good agreement to the first-phase precipitation of $\mathrm{DO}_{22}$ at a 4 at.\% $\mathrm{Al}$ alloy(Figure 2(a)). The final equilibrium volume fraction of $\mathrm{DO}_{22}$ is larger than that of the $\mathrm{L}_{2}$ phase (Figure $3(\mathrm{a})$ ), which suggests that $\mathrm{DO}_{22}$ is dominated the $\mathrm{Ni}_{75} \mathrm{Al}_{4} \mathrm{~V}_{21}$ system. And this also is testified by microstructure morphology in Figure 2(d). With respect to a 6 at.\% $\mathrm{Al}$ alloy (Figure 3(b)), the volume fractions of $\mathrm{DO}_{22}$ and $\mathrm{L}_{2}$ increase simultaneously and rapidly indicating the drastically growth and compotation of the two ordered phases, and the two approach equilibrium rapidly with time proceeds. This phenomenon is consistent with the microstructure morphology at the 6 at.\% Al alloy (Figure 2(b) and 2(e)). When the $\mathrm{Al}$ concentration is enhanced till 8 at.\%, the volume fraction of $\mathrm{L}_{2}$ increases quickly at the initial stage, and grows quickly into a stable ordered phase, then finally approaches equilibrium (Figure 3(c)), all of which is in line with microstructure morphology within the same concentration (Figure 2(c)). The final equilibrium values were approximately the same for the two phases, which is in agreement with the results in Figure 2(f).

\section{Discussion}

The interatomic potentials curves (Figure 4) are applied to investigate the variations of precipitation sequence with $\mathrm{Al}$ concentration ranging from 1 to 21 at. $\%$. The Ni-Al interatomic potentials increase in an approximately linear manner with enhanced Al level, at constant $1000 \mathrm{~K}$ (solid line, Fig- 

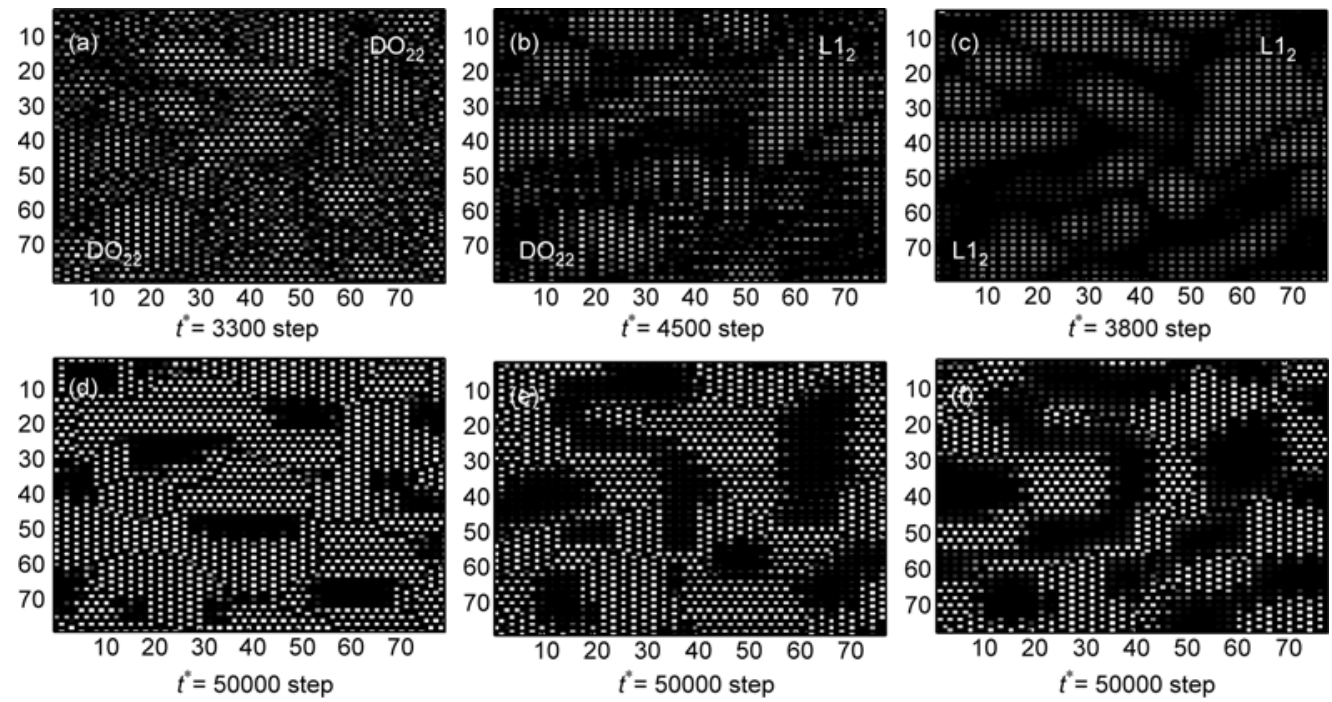

Figure 2 Variation of interatomic potentials with $\mathrm{Al}$ concentration $\left(c_{\mathrm{Al}}\right)$ and time step $\left(t^{*}\right)$. (a), (d) $c_{\mathrm{Al}}=4$ at. $\%,(\mathrm{~b}),(\mathrm{e}) c_{\mathrm{Al}}=6$ at. $\%$ and (c), (f) $c_{\mathrm{Al}}=8$ at. $\%$.
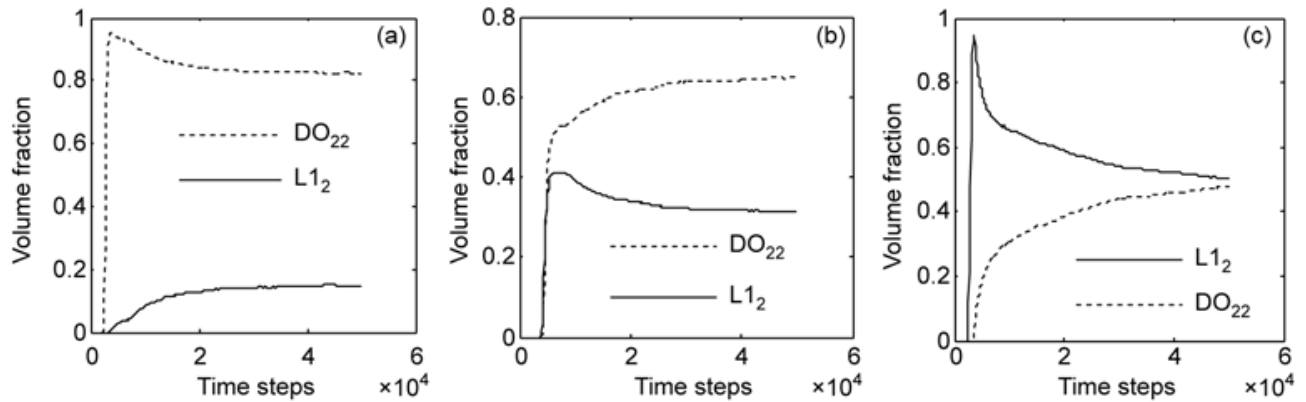

Figure 3 Volume fraction of $\mathrm{Ni}_{75} \mathrm{Al}_{x} \mathrm{~V}_{25-x}$ alloy as a function of $\mathrm{Al}$ concentrations (a) 4, (b) 6 and (c) 8 at. $\%$.
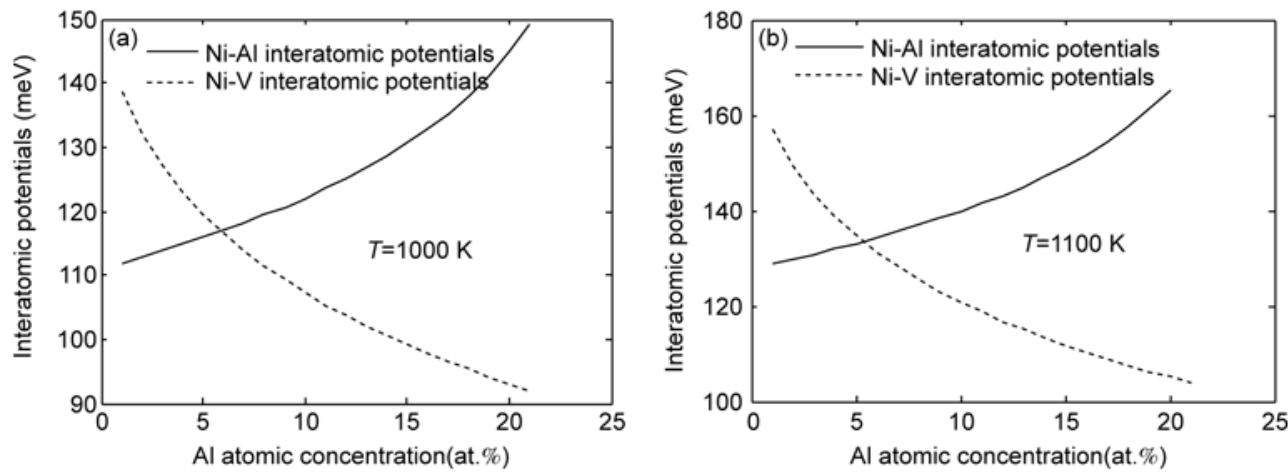

Figure 4 Variation of interatomic potentials with atomic concentrations at (a) 1000 and (b) $1100 \mathrm{~K}$.

ure 4). Similarly, the Ni-V interatomic potentials decrease linearly with enhanced Al level (dashed line, Figure 4). The two curves have a intersection at 6 at.\% $\mathrm{Al}$ alloy, where the interatomic potentials of $\mathrm{Ni}-\mathrm{Al}$ equal to those of $\mathrm{Ni}-\mathrm{V}$. On the left side of this intersection, the dashed line is greater than the solid line, which implied the interatomic potentials of $\mathrm{Ni}-\mathrm{Al}$ were larger than those of $\mathrm{Ni}-\mathrm{V}$. On the right of the intersection, the solid line is greater than the dashed line, which indicates the interatomic potentials of $\mathrm{Ni}-\mathrm{V}$ are larger than those of Ni-Al. Similar curves were obtained at concentrations range from 1 at. $\%$ to 21 at. $\%$, at $1100 \mathrm{~K}$ (Figure 4(b)). The two curves intersect at a different concentration ( 5 at.\% Al) with that of $1000 \mathrm{~K}$, however. For this case, the interatomic potentials of $\mathrm{Ni}-\mathrm{Al}$ and $\mathrm{Ni}-\mathrm{V}$ are equal at the 5 at.\% $\mathrm{Al}$ alloy. With the enhanced $\mathrm{Al}$ level, the Ni-Al interatomic potentials increase while $\mathrm{Ni}-\mathrm{V}$ interatomic potentials decrease, individually.

Based on the above analysis, the following precipitation 
patterns are evident: the first precipitated phase is $\mathrm{DO}_{22}$ (Figure 2(a)) for a 4 at.\% $\mathrm{Al}$ alloy, which is contributed to the higher interatomic potentials of $\mathrm{Ni}-\mathrm{V}$ than those of $\mathrm{Ni}$-Al (Figure 4(a)). $\mathrm{L}_{2}$ and $\mathrm{DO}_{22}$ phases precipitate simultaneously for 6 at.\% Al alloy (Figure 2(b)), which occur due to the equality of the interatomic potentials of the two phases (Figure 4(a)). The $\mathrm{L} 1_{2}$ phase precipitates first for the 8 at.\% Al alloy(Figure 2(c)) attributing to the interatomic potentials of Ni-Al are higher than those of Ni-V (Figure 4(a)). In summary, the precipitation sequences for the two phases were closely associated with the interatomic potentials.

\section{Conclusions}

The equations for interatomic potentials for $\mathrm{L}_{2}$ and $\mathrm{DO}_{22}$ phases are formulated according to the relationship equations proposed by Khachaturyan. These equations are then applied to computed the interatomic potentials of Ni-Al $\left(\mathrm{L}_{2}\right.$ phase) and $\mathrm{Ni}-\mathrm{V}\left(\mathrm{DO}_{22}\right.$ phase) with varying temperature and concentration. The computed results are in good agreement with the experimental results, which verifies the reliability of the results.

Both interatomic potentials vary in an approximately linear manner with enhanced $\mathrm{Al}$ level, which the interatomic potentials for $\mathrm{Ni}-\mathrm{Al}$ increase but those for $\mathrm{Ni}-\mathrm{V}$ decrease. The microstructure morphology and temporal evolutional volume fraction for the two phases are obtained by substituting the interatomic potentials into microscopic phase field theory, which shows that the competitive precipitation sequence of the phases relate to the interatomic potentials closely. The $\mathrm{Ni}-\mathrm{V}$ interatomic potentials are larger than those of $\mathrm{Ni}-\mathrm{Al}$ when the $\mathrm{Al}$ concentration is smaller than 6 at. $\%$, and $\mathrm{DO}_{22}$ phase precipitates first and incubates shorter than the $\mathrm{L}_{2}$ phase. The $\mathrm{Ni}$ - $\mathrm{Al}$ interatomic potentials are larger than those of $\mathrm{Ni}-\mathrm{Al}$ when the $\mathrm{Al}$ concentration is greater than 6 at. $\%$, and $\mathrm{L}_{2}$ phase precipitates first with a shorter gestation stage than $\mathrm{DO}_{22}$. At 6 at.\% $\mathrm{Al}$ concentration, the interatomic potentials are equal leading to the two phases simultaneous precipitation of $\mathrm{L}_{2}$ and $\mathrm{DO}_{22}$ phases.

This work was supported by the National Natural Science Foundation of China (10902086, 51075335 and 50875217), the Basic Research Fund of the Northwestern Polytechnical University (JC201005) and the Doctoral Foundation of Northwestern Polytechnical University.

1 Li L, Lu X Y, Cao C D, et al. Solidification microstructure and phase constitution of $\mathrm{Fe}-7.5 \% \mathrm{Mo}-16.5 \% \mathrm{Si}$ ternary quasiperitectic alloy (in Chinese). Chinese Sci Bull, 2009, 54: 2108-2112

2 Pareige C, Blavette D. Simulation of the $\mathrm{FCC} \rightarrow \mathrm{FCC}+\mathrm{Ll}_{2}+\mathrm{DO}_{22}$ kinetic reaction. Scrip Mater, 2001, 44: 243-247

3 Poduri R, Chen L Q. Computer simulation of atomic ordering and

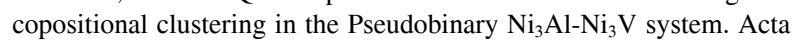
Mater, 1998, 46: 1719-1729

4 Zapolsky H, Pareige C, Marteau L, et al. Atom probe analyses and numerical calculation of ternary phase diagram in Ni-Al-V system. Calphad, 2001, 25: 125-134

5 Li Y S, Chen Z, Lu Y L, et al. Microscopic Phase-Field simulation of coarsening behavior of precipitates in $\mathrm{Ni}_{75} \mathrm{Al}_{x} \mathrm{~V}_{25-x}$ alloy (in Chinese). Acta Metall Sin, 2006, 42: 239-244

6 Li Y S, Chen Z, Lu Y L, et al. Microscopic phase-field simulation for nucleation incubation time of $\mathrm{Ni}_{75} \mathrm{Al}_{x} \mathrm{~V}_{25-x}$ alloy. J Cent South Univ Technol, 2005, 12: 635-640

7 Hou H, Zhao Y H, Chen Z, et al. Prediction for the early precipitation progress of $\mathrm{Ni}_{75} \mathrm{Al}_{x} \mathrm{~V}_{25-x}$ system with lower $\mathrm{Al}$ concentration by the Phase-Field model (in Chinese). Acta Metall Sin, 2005, 41: 695-702

$8 \mathrm{Hu} \mathrm{D}$, Huang A J, Wu X. On the massive phase transformation regime in TiAl alloys: The alloying effect on massive/lamellar competition. Intermetallics, 2007, 15: 327-332

9 Li J Q, Tang Y L, Shen N F, et al. Effects of solidification kinetics on phase selection of Ni-Al alloys. J Alloy Compd, 2001, 329: 157-161

10 Liu Y C, Yang G, Zhou Y H. Phase competition in the undercooled hypoperitectic $\mathrm{Ti}_{47} \mathrm{Al}_{53}$ alloy. Mater Lett, 2002, 57: 315-324

11 Kim H K, Jung W S, Lee B J. Modified embedded-atom method interatomic potentials for the Fe-Ti-C and Fe-Ti-N ternary systems. Acta Mater, 2009, 57: 3140-3147

12 Pasianot R C, Malerba L. Interatomic potentials consistent with thermodynamics: The Fe-Cu system. J Nucl Mater, 2007, 360: 118-127

13 Liubich V, Dorfman S, Fuks D. Non-empirical study of phase competition in A2-B2 mixture in Fe-Al alloy. Comput Mater Sci, 2002, 24: $268-272$

14 Khachaturyan A G. Theory of Structural Transformation in Solids. New York: Wiley, 1983

Open Access This article is distributed under the terms of the Creative Commons Attribution License which permits any use, distribution, and reproduction in any medium, provided the original author(s) and source are credited. 